\title{
Stellar Populations in Spiral Galaxies
}

\author{
Lauren A. MacArthur ${ }^{1}$, Jesús J. González ${ }^{2}$ and Stéphane Courteau ${ }^{3}$ \\ ${ }^{1}$ California Institute of Technology, Pasadena CA 91125, USA \\ email: lam@astro.caltech.edu \\ ${ }^{2}$ Instituto de Astronomía, UNAM, Mexico \\ ${ }^{3}$ Queen's University, Kingston, ON K7L 3N6, Canada
}

\begin{abstract}
We report preliminary results of the characterization of bulge and inner disk stellar populations for 8 nearby spiral galaxies using Gemini/GMOS. The long-slit spectra extend out to $1-2$ disk scale lengths with $\mathrm{S} / \mathrm{N} / \AA ̊>50$. Two different model fitting techniques, absorptionline indices and full spectral synthesis, are found to weigh age, metallicity, and abundance ratios differently, but with careful attention to the data/model matching (resolution \& flux calibration), we are able constrain real signatures of age and metallicity gradients in star-forming galaxies.
\end{abstract}

Keywords. galaxies: abundances, galaxies: spiral, galaxies: formation

\section{Introduction}

Understanding the formation and evolution of disk galaxies, in particular the formation of their spheroidal components, remains a challenge both from the theoretical and observational perspectives. Traditional views suggest that all bulges formed as "miniature ellipticals" via dissipationless collapse (e.g. Eggen et al. 1962) or by the violent merging of smaller fragments (e.g. Kauffmann 1996) at high-redshift. A robust prediction from these models is that all spheroids have old stellar populations (SPs), with bulges being older than field ellipticals at a given redshift. These views have been challenged by recent observations suggesting ongoing formation of galactic bulges due, perhaps, to a redistribution of disk material from the presence of a bar (Kormendy \& Kennicutt 2004).

Detailed analysis of the SPs in galaxies can provide invaluable clues about their formation and subsequent evolution. Broad-band colors have often been used as a proxy for SP parameters (e.g. MacArthur et al. 2004). Color-based studies, however, are plagued by a degeneracy between the effects of age, metallicity $(Z)$, and dust reddening, all leading to redder colors. Spectroscopic techniques, largely impervious to dust effects (MacArthur 2005), offer a more detailed and discriminating view, especially in light of the latest implementations of SP synthesis models (e.g. Vazdekis 1999; Bruzual \& Charlot 2003).

Existing spectroscopic studies of bulges spanning the full range of Hubble types are few, and the results are conflicting. The absorption-line studies of the central regions of $\mathrm{Sa}-\mathrm{Sc}$ spirals of Trager et al. (1999) and Proctor \& Sansom (2002, PS02) both find that latetype bulges cannot be reproduced using primordial collapse models and invoke extended gas infall to explain the observations, while, in a similar study, Goudfrooij et al. (1999) conclude the opposite. Recently, Thomas \& Davies (2006) reanalyzed the PS02 data and found no difference between the SPs of spiral bulges and Es at a given central velocity dispersion, $\sigma_{0}$, concluding that processes involving disk material cannot be responsible for the recent star formation implied by the young ages. A significant outlier, however, is the bulge of our own Milky Way (MW), studies of which reveal old and $\alpha$-enhanced SPs that must have formed long ago and on short timescales. This observation is difficult to reconcile with the MW's small $\sigma_{0}$ and the presence of a bar. As Thomas \& Davies 
point out, the MW studies sample a larger physical radius than those of external galaxies so this conundrum could be resolved if there is a positive age gradient in the bulge. At the heart of these conflicts is the fact that extant studies of local bulges are limited in one way or another (sample size, range in Hubble type, data of insufficient depth and/or spatial resolution for studies of gradients and assessment of disk contamination, etc.)

\section{Program and Observations}

To address the above issues, and to compare different techniques for measuring SP parameters for composite systems, we have obtained Gemini/GMOS long-slit spectroscopy for a pilot sample of 8 nearby face-on field spirals ( $\mathrm{Sa}-\mathrm{Scd}$ ) from which we measure a suite of absorption line-indices and perform full spectral synthesis fits through the bulges and inner disks. Our simultaneous spectral coverage of $\sim 4050-6750 \AA$ includes most of the major atomic and molecular features to disentangle age \& $Z$ effects in integrated galaxy spectra. Integration times of 45-72 min per galaxy were required to achieve adequate $\mathrm{S} / \mathrm{N} / \AA(>50)$ into the disk, to constrain disk populations and to allow for the removal of disk contamination in the bulge. Details about the data and reductions, including calibration ( $\lambda$ \& flux), resolution (instrumental \& profiles), rotation, velocity dispersion, and bulge/disk decontamination will be described in González et al. (2007, in prep.).

\section{Stellar Population Indicators}

\subsection{Lick Indices}

As a first attempt at constraining the SPs in our galaxies, we measured 24 spectral (Lick/IDS) indices (missing only the bluest $\mathrm{H} \delta_{A}$ ), and compared them with the SSP models of BC03. Many of our galaxies have significant line-emission, some extending all the way into the center. Many authors attempt to correct for emission by fitting emissionfree templates to their galaxy spectra (e.g. González 1993). However, we are interested here in testing the discriminating power of the indices on their own (i.e. without the need for accompanying higher-resolution spectra). We thus did not apply any corrections to individual indices, but rather used a scheme in which the emission-line affected indices are systematically eliminated from the fit of indices to SSP models.

By comparing the age \& $Z$ measurements using different index combinations, depending on the pathologies of the given spectrum, we conclude that the metallic indicators are fairly reliable but, when emission is present, there is simply not enough sensitivity in the indices unaffected by emission to obtain reliable age estimates.

\subsection{Spectral Fitting}

We thus turned our attention to full spectral synthesis fits. While these offer the advantage of being able to mask out undesirable regions of the spectrum, the wider wavelengthbaseline reinforces the sensitivity to dust extinction along with fluxing and resolution issues (which must be carefully matched between data and models). Our spectral synthesis technique is an optimized linear, bound-constrained (non-zero), combination of BC03 SSP model templates (spanning ages 0.001-20Gyr and $\left.Z=(0.02-2.5) Z_{\odot}\right)$, while masking out regions affected by emission, persistent sky lines, and CCD gaps. Best-fit SSP and mixed populations were obtained for each radial bin of each galaxy. Fig. 1 shows an example of our full synthesis fits for a spectrum showing strong emission. The data-model agreement is quite remarkable throughout the entire spectrum. Analysis of the radial bins for each galaxy also demonstrated the stability of our full spectral fitting technique, whereas the SSP fits were unstable producing spurious gradients. 


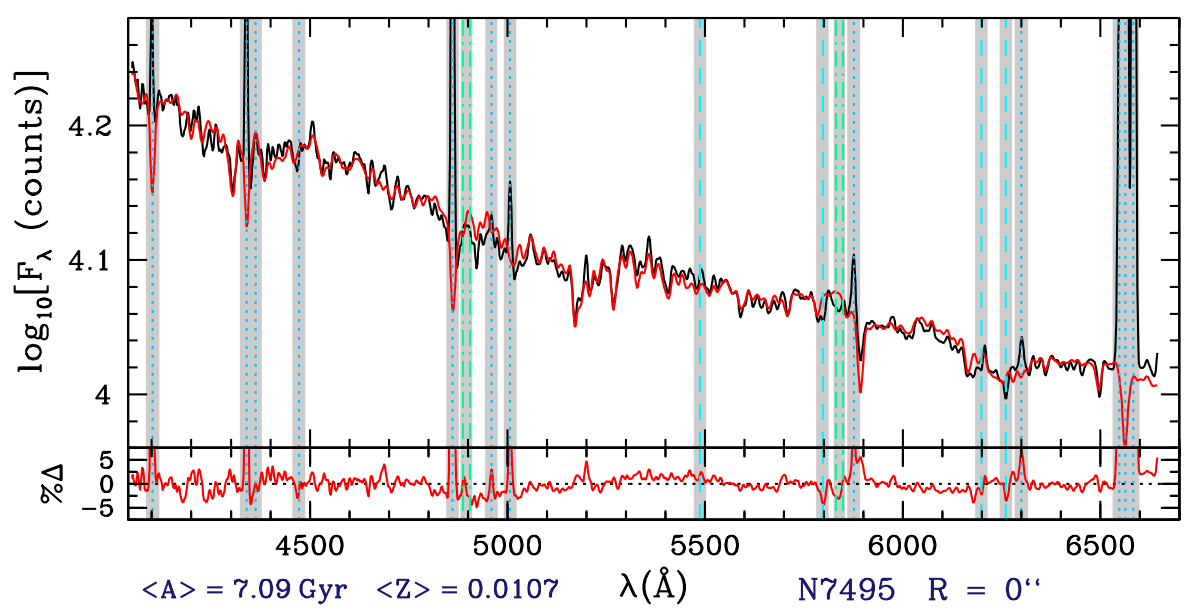

Figure 1. Full spectral synthesis fit to the central spectrum of the emission-line dominated Sc galaxy NGC 7495. Black line: data. Red line: model fit. Shading indicates emission-line regions (including the Balmer series, [O III], [N II], etc., are indicated by vertical dotted blue lines), variable night sky lines (dashed light blue lines), and CCD gaps (dashed light green line), that are excluded from the fit. Residuals are shown as percent galaxy-model differences.

Our study is the first to provide radially resolved spectra well into galaxy disks, enabling a comparison of the bulge and inner disk populations. Fig. 2] shows two examples of the radial profiles of average age \& $Z$ as determined from our full spectral synthesis fits. Of particular note is the spike to older ages just beyond $\sim 1 r_{e}$ for NGC 628 . This is precisely the signature noted in $\$ 1$ required to reconcile the discrepant results for the MW bulge. The fact that we observe this signature on both sides of the galaxy (whose spectra were fit entirely independently) renders confidence that this signature is real $\rightarrow$ While the small size of our sample does not allow for a detailed statistical study of galaxy parameters with inferred age \& $Z$, several important observations emerge: (1) late-type bulges show recent star formation in their centers, potentially driven by secular evolution processes; (2) most bulges show positive age gradients (younger toward the center) and negative $Z$ gradients; and (3) inner disks show weak age gradients (if any) and all have significant negative or flat radial $Z$ profiles. We must caution that to truly assert the reality of young bulge SPs requires the careful removal of any contamination of disk light. This is indeed a major focus of our continued investigation of this rich data set.

\section{Summary}

Using radially resolved long-slit spectra of 8 star-forming galaxies, we have assessed the strengths and weaknesses of different SP analysis techniques in the presence of emission lines. The most pertinent observations are as follows:

- With moderate spectral resolution, good $\lambda$ coverage, and high $\mathrm{S} / \mathrm{N} / \AA(>50)$, measurement of light-weighted ages \& metallicities for star-forming galaxies is feasible.

- Details are critical: calibration ( $\lambda$ \& relative flux), resolution, velocity dispersion, and rotation must be treated self-consistently between the data and models.

- Different fitting techniques weigh age, metallicity, and abundance ratios differently: Balmer emission limits age fitting from indices; age information is recovered (in the

$\dagger$ Using SAURON observations, Gandia et al. (in prep.) observe the same trend in age for NGC 628 using a fully independent approach. 

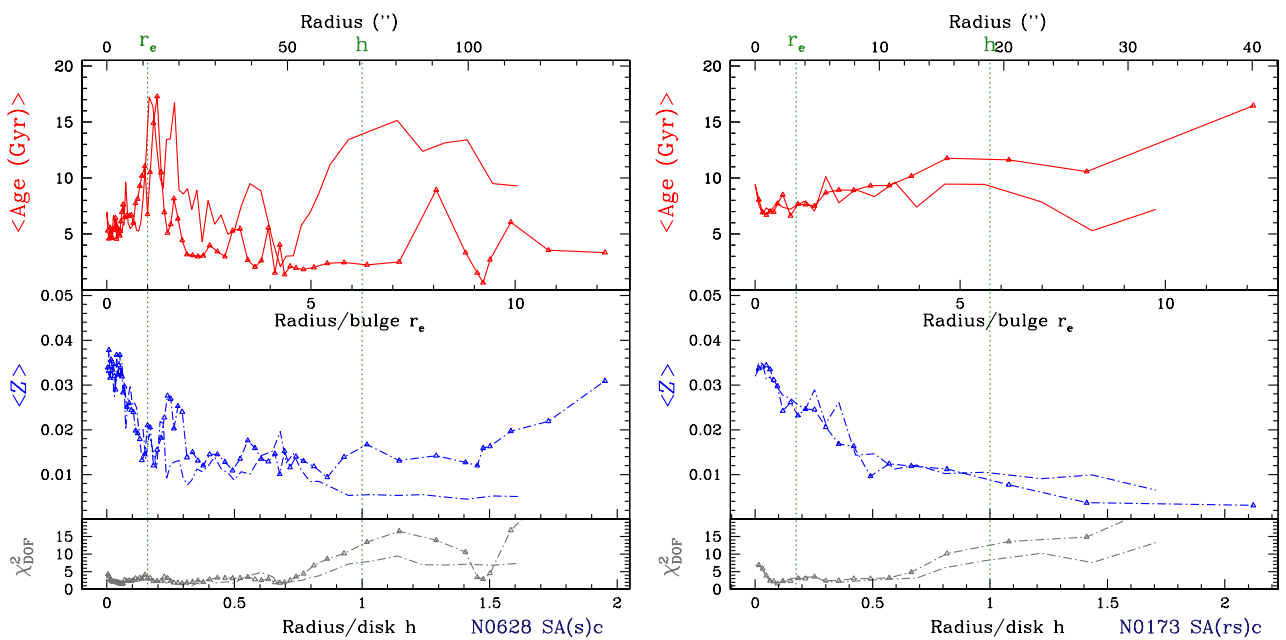

Figure 2. Light-weighted gradients in average age (top panels) and metallicity (middle panels) from full spectral synthesis fits. The reduced $\chi^{2}$ from the fit is shown in the bottom panels. The two distributions in each panel indicate the two sides of the galaxy with the common side marked by triangles. Vertical dotted green lines mark the bulge effective (half-light) radius, $r_{e}$, and 1 disk scale length, $h$.

presence of emission) from full spectrum and continuum SED fitting (but compounds the caveats about dust extinction and fluxing that are less important for indices).

- SSPs are not a good match to late-type galaxies. The degeneracies between age, metallicity, dust, etc. are extreme leading to unstable fits.

- Full spectral synthesis fitting is consistent with metallicity measured via indices, but is far less sensitive to abundance variations.

Ultimately, we desire a large enough sample to look for trends with galaxy parameters allowing for the discrimination between different scenarios for current models of galaxy formation and to guide future implementations of such models.

\section{Acknowledgements}

We would like to thank the conference organizers for putting together such a stimulating and informative meeting.

\section{References}

Bruzual, A. G. \& Charlot, S. 2003, MNRAS, 344, 1000 [BC03]

Eggen, O. J., Lynden-Bell, D., \& Sandage, A. R. 1962, ApJ, 136, 748

González, J. J. 1993, Ph.D. thesis, Univ. California, Santa Cruz

Goudfrooij, P., Gorgas, J., \& Jablonka, P. 1999, Ap\&SS, 269, 109

Kauffmann, G. 1996, MNRAS, 281, 487

Kormendy, J., \& Kennicutt, R. C., Jr. 2004, ARA\&A, 42, 603

MacArthur, L. A., Courteau, S., Bell, E., \& Holtzman, J. A. 2004, ApJS, 152, 175

MacArthur, L. A. 2005, ApJ, 623, 795

Proctor, R. N., \& Sansom, A. E. 2002, MNRAS, 333, 517 [PS02]

Thomas, D., \& Davies, R. L. 2006, MNRAS, 366, 510

Trager, S. C., Dalcanton, J. J., \& Weiner, B. J. 1999, The Formation of Galactic Bulges, eds.

Carollo, Ferguson, \& Wyse, Cambridge Univ. Press, p42

Vazdekis, A. 1999, ApJ, 513, 224 